\title{
苦草繁殖生态学研究
}

\section{陈开宁 $1^{12}$ 兰策介 ${ }^{1} \quad$ 史龙新 $^{3} \quad$ 陈伟民 ${ }^{1}$ 许 海 $^{1} \quad$ 包先明 $1^{12}$}

(1 中国科学院南京地理与湖泊研究所, 南京 210008) (2 中国科学院研究生院 北京 100039)

（3 无锡市太湖湖泊治理有限责任公司，江苏无锡 214023)

摘 要 通过太湖野外调查、室内培养、种子与块茎萌发及幼苗生长试验, 对太湖苦草 ( Vallisneria natans)种群的繁 殖生态特征进行了研究。结果显示: : 1) 苦草分配于有性繁殖部分的生物量较无性的大, 分别占总生物量的 $25.0 \%$ $\pm 13.8 \%$ 和 $10.1 \% \pm 7.0 \%$ 。雌花数与座果率平均分别为 $22.9 \pm 13.8$ 朵 株 ${ }^{-1}$ 和 $73.3 \% \pm 17.9 \%$ 。雄株可以产生 11 33 个佛焰苍, 每个佛焰苍内平均含有 $364 \pm 38$ 朵雄花, 每个雄花产生的花粉为 $128 \sim 184$ 粒。每个果实内种子 丰富，多达 $150 \sim 360$ 粒，估算太湖苦草种群立地种子量可达 $1.68 \times 10^{4} \sim 1.01 \times 10^{6}$ 个 $\mathrm{m}^{-2}$ 。但每年种群主要来自 地下块茎和匍匐茎, 水深、风浪等可能是种子苗难以在湖泊中定植成功的主要因素。2)苦草的块茎数量较大, 平均 90 226 个 $\mathrm{m}^{-2}$, 是种群发展的强大物质基础。3)温度、光照、基质及种子保存方式与时间长短对种子发芽率均有 较大影响。10 ${ }^{\circ} \mathrm{C}$ 时种子发芽率较低, 仅 $8.35 \% \pm 1.89 \%, 20{ }^{\circ} \mathrm{C}$ 时发芽率较高, 为 $56.73 \% \pm 6.42 \%, 30{ }^{\circ} \mathrm{C}$ 时发芽率有 所降低, 为 $43.55 \% \pm 4.34 \%$ 。种子发芽对光照有一定要求, $20{ }^{\circ} \mathrm{C}$ 、无光条件下 种子发芽率下降 $63.6 \%$ 。在没有湖 泥为基质的情况下 发芽率下降 36.5\%。此外, 随干燥保存时间增加 种子发芽率下降。4)块茎发芽对光照需求不 大, 但温度对块茎的发芽率有较大影响, $10{ }^{\circ} \mathrm{C}$ 时发芽率为 $20.3 \% \pm 5.7 \%, 20{ }^{\circ} \mathrm{C}$ 时较高, 达 $90.2 \% \pm 12.6 \%, 30{ }^{\circ} \mathrm{C}$ 时 发芽率降低至 $60.4 \% \pm 7.6 \%$ 。5) 光照对幼苗生长有一定影响。有光照的幼苗伸长生长比无光条件下慢, 但生物 量积累较大, 有光的苗生长速度平均为 $0.56 \sim 0.70 \mathrm{~cm} \mathrm{~d}^{-1}$, 无光的为 $0.86 \sim 0.96 \mathrm{~cm} \mathrm{~d}^{-1}$ 。试验结果还显示, 苦草 的块茎苗初期的伸长生长主要依赖于基部的根茎生长。6)根据研究结果认为, 在湖泊中恢复苦草种群应主要利用 地下块茎 种子只适用于相对静止的浅水池塘种植。

关键词 种子 块茎 发芽率 苦草 恢复 沉水植物

\section{REPRODUCTIVE ECOLOGY OF VALLISNERIA NATANS}

\author{
CHEN Kai-Ning $^{1}{ }^{2}$ LAN Ce-Jie ${ }^{1}$ SHI Long-Xin ${ }^{3}$ CHEN Wei-Min ${ }^{1} \quad$ XU Hai $^{1}$ and BAO Xian-Ming ${ }^{1}{ }^{2}$ \\ (1 Nanjing Institute of Geography and Limnology, Chinese Academy of Sciences , Nanjing 210008 , China) \\ (2 Graduate School of Chinese Academy of Sciences, Beijing 100039, China) \\ (3 Wuxi Taihu Lake Management CO . , LTD. , Wuxi, Jiangsu 214023 , China)
}

\begin{abstract}
Vallisneria natans is a dominant submerged macrophyte in Lake Taihu. The biomass and quantity of propagules of $V$. natans were investigated in Lake Taihu in October , 2003 and in March , 2004. Seed and tuber germination, and development of seedlings produced from tubers under different conditions were also studied in the laboratory. Our objectives were to understand characteristics of the reproductive ecology in $V$. natans. The results showed that the biomass of asexual and sexual propagules was $10.1 \% \pm 7.0 \%$ and $25.0 \% \pm 13.8 \%$ of total biomass , respectively , and the biomass of sexual propagules was greater than asexual propagules. The average number of female flowers was $22.9 \pm 13.8$ per individual plant, and seedset rate was $73.3 \% \pm 17.9 \%$. There were $11-33$ spathes per individual male plant and $364 \pm 38$ male flowers inside each spathe. Based on our in situ investigation, we estimated a density of $6.29 \times 10^{5}-1.89 \times 10^{6}$ microspores $\mathrm{m}^{-2}$ around $V$. natans populations in Lake Taihu. Seedlings produced from seeds were not able to survive in Lake Taihu, which is likely caused by factors such as wind, wave and water depth. Most populations in $V$. natans reproduced asexually from root tubers. We estimated a mean tuber density of $90-226$ per square meter. Light, temperature, sediment, thermal fluctuation and seed age had a marked influence on germination rates. Germination rates of seeds stored for 4 months under water in the lake was $8.35 \% \pm 1.89 \%$, $56.73 \% \pm 6.42 \%$ and $43.55 \% \pm 4.34 \%$ at 10,20 and $30{ }^{\circ} \mathrm{C}$, respectively. Seed germination rates stored for one year was $35.53 \% \pm 3.53 \%$ under natural light conditions and decreased to $12.63 \% \pm 2.86 \%$ under dark conditions. Germination rates of seeds stored for one or two years was $35.53 \% \pm 3.53 \%$ and $12.63 \pm$
\end{abstract}


$2.86 \%$, respectively. Germination rates of seeds stored for four years was near zero. Germination rates of seeds decreased under conditions without sediment. Tubers did not require light for germination. There was an optimal temperature of $20{ }^{\circ} \mathrm{C}$ for tuber germination to occur that was as high as $90.2 \% \pm 12.6 \%$. Biomass accumulation in seedlings produced from tubers under light conditions was greater than that in the dark, while leaves of seedlings grown in the dark were longer. Seedling increases in length mainly depends on the stems of the lower end of leaves. We present suggestions for restoration efforts of populations of $V$. natans in Lake Taihu.

Key words Seed, Tuber, Germination, Vallisneria natans, Restoration, Submerged macrophyte

苦草( Vallisneria natans)，俗名面条草、扁担草、 水非菜等, 属水鳖科苦草属多年生沉水植物, 为我国 最常见的沉水植物之一。依据《中国植物志》第 8 卷 (孙祥钟,1992) 和《中国水生高等植物图说》(颜素 珠, 1983) 记载, 我国苦草属植物有苦草、刺苦草 $(V$. spinulos $a)$ 、密刺苦草 $(V$. denseserrulata $)$ 和亚洲苦草 $(V$. asiatica $)$,但没有苦草 $(V$. spiralis $)$ 的记载。

据研究, 苦草生态适应性广吸附污物及营养盐 能力强, 是减少水体污染, 缓解水体富营养化程度的 重要沉水植物(宋福等, 1997)。苦草繁殖速度快, 再 生能力强, 刏割后恢复时间短, 被刏割的苦草仅 $15 \mathrm{~d}$ 就可恢复生长到收割前水平(顾林娣等, 1994) ,可从 水体中带出大量营养盐。因此, 苦草常被作为沉水 植物恢复主耀选用品种之一。

从已有的文献看, 我国苦草属植物的研究主要 以分类学为主, 有关苦草生态学特征的认识多散见 于以水生植物调查为主要目的的文献中, 而针对苦 草繁殖的生态特征的研究则较少, 许多生物学一般 性的描述主要见于各类植物志与教材中。近年来， 有苦草种子与块茎 (冬芽、球茎) 发芽生态方面的报 道。由文辉和宋永昌 (1995) 研究了淀山湖苦草 $(V$. spiralis) 种子的萌发生态, 发现干燥保存数月的苦草 种子萌发最适温度为 $20{ }^{\circ} \mathrm{C}$, 最适基质为土 + 水。熊 秉红和李伟 (2000) 对鄱阳湖的苦草种子进行了萌发 实验, 发现新鲜种子萌发的最适温度是 $25{ }^{\circ} \mathrm{C}$, 最适 基质为沙质。李亚东和崔艳秋 (2000)的研究显示, 武汉东湖干燥保存数月的苦草种子和块茎发芽最适 温度为 $20{ }^{\circ} \mathrm{C}$ 种子和块茎的发芽最适基质是水 + 湖 泥 3 种基质里发芽率的顺序为水 + 湖泥 $>$ 水 + 沙 $>$ 水。显然, 文献报道存在一定差异, 对这方面的认 识仍然需要更多的试验数据来加以充实。

针对上述问题，笔者对太湖的苦草繁殖生态进 行了野外调查与观察, 并对种子保存时间、基质条 件、温度、光照对种子和地下块茎发芽率影响, 以及 光照对幼苗生长影响进行了研究, 目的是深入了解 苦草在自然界中繁殖特性与每年种群恢复物质基
础, 进一步研究种子及块茎萌发生态, 同时, 也为浅 水湖泊沉水植被的恢复提供理论依据。

\section{1 研究方法}

\section{1 野外调查与试验材料采集}

考虑 10 月的苦草种群数量大, 同时为繁殖生长 旺期 果实和地下块茎数量大 3 月是种群恢复与繁 殖体萌发期, 因此, 分别于 2003 年 10 月和 2004 年 3 月, 对太湖地区苦草种群分布进行了野外调查 (由于 此文主题在于苦草繁殖方面, 种群分布将另作报 道), 同时对苦草的繁殖生态特性 (开花数、座果数、 繁殖部分生物量、有性繁殖部分生物量、地下块茎在 底泥的埋藏深度、数量及生物量) 进行现场采样观 察, 并用数码照相机拍照。然后将采集的块茎和果 实带回实验室, 观测苦草种子和球茎在试验条件下 的发芽率、幼苗生长情况。

太湖苦草种群主要分布于贡湖湾东部及南部湾 口、镇湖湾、胥口湾南部、胥口湾与东太湖连通的河 道、东太湖 (庙港、渡村、戗港、横泾附近) 、太湖南部 的七都附近至东太湖水域。试验观察的苦草样品采 集于上述水域。

\section{2 室内试验}

\subsection{1 种子发芽试验}

受试种子共有 4 种, 1 种是从太湖采集果实, 保 存在太湖野外实验基地湖水中, 试验前取回试验室 ; 另外 3 种是太湖采集的果实, 干燥保存, 受试种子保 存时间为 $1 、 2 、 4$ 年。这 4 种种子与太湖采集的当年 块茎进行同期的发芽试验。首先, 将种子的果实放 入烧杯中用蒸馏水浸泡 $48 \mathrm{~h}$ 然后用手将果荚揉碎, 让种子游离出来。由于种子较小, 用胶头滴管数 1000 粒, 再将种子均匀地移到试验瓶中进行发芽试 验。受试种子共分 10 组, 每组设 3 个重复。处理 1 、 $2 、 3$ 组分别是在温度为 $10 、 20 、 30{ }^{\circ} \mathrm{C}$ 条件下, 于装有 $2 \mathrm{~cm}$ 厚湖泥作为基质的 $500 \mathrm{ml}$ 烧杯中进行发芽试 验沚理 4 组为无湖泥的 $20{ }^{\circ} \mathrm{C}$ 条件下的发芽试验; 处理 5.6 组分别为有 $2 \mathrm{~cm}$ 厚湖泥作为基质, 于 $30 /$ 
$10 、 20 / 10{ }^{\circ} \mathrm{C}$ 变温条件下的发芽试验 处理 $1 \sim 6$ 组用 的都是当年的种子在自然光下进行的 (中午照度约 为 $350 \sim 2600 \mathrm{Lux}$ )。处理 7 组用干燥保存 1 年的种 子, 在自然光下进行发芽试验; 处理 8 组用干燥保存 1 年的种子做无光发芽试验; 另外两组分别用干燥 保存 $2 、 4$ 年的种子在自然光下进行发芽试验。处理 $7 \sim 10$ 组试验温度为 $20{ }^{\circ} \mathrm{C}$, 有 $2 \mathrm{~cm}$ 厚湖泥作为基 质。上述所有试验用的烧杯内有 $8 \mathrm{~cm}$ 高的蒸馏水。 试验时间为 3 月 26 日至 6 月 19 日。试验结束时， 将苦草幼苗连同湖泥一起倒在托盘上, 数发芽数, 计 算发芽率。

\section{2 .2 球茎发芽试验}

受试块茎是从东太湖戗港附近 $\left(120^{\circ} 49.458^{\prime} \mathrm{E}\right.$, $31^{\circ} 07.895^{\prime} \mathrm{N}$ ) 采集而来。试验时先将块茎洗干净, 再用吸水纸吸干, 逐个称其重量并编号; 同时, 取 8 个烧杯, 杯底铺 $2 \mathrm{~cm}$ 经过筛选的松土, 然后按编号 将块茎沿杯壁依次放在土上, 再在上面铺 $0.5 \mathrm{~cm}$ 用 水清洗过的细沙, 最后加入高为 $8 \mathrm{~cm}$ 水, 加水时让 水缓慢的沿着杯壁流入，以免扰动杯底的沙和土。 试验共 2 组, 每组设 4 个重复。 1 组做有光试验, 直 接放入试验室; 另 1 组做无光试验, 用黑桶套住, 再 用纸和黑方便袋封住, 再放入培养室。培养条件 温 度 $20{ }^{\circ} \mathrm{C}$, 光照 $2200 \mathrm{Lux} 、 12 / 24 \mathrm{~h}$, 时间 3 月 26 日至 4 月 10 日。试验结束时, 将苦草植株按编号顺序取出 放在盛有水的托盘上, 然后逐个吸干称重, 量其株 高、茎长、叶长和叶宽, 及无光条件下苦草植株所生 长的高度。

\section{3 数据处理}

用 SigmaPlot 8.0 软件处理、分析数据差异 并作图。

\section{2 实验结果}

\section{1 生物学与繁殖生态学特性}

苦草叶长带形基生, 叶最长可达 $2 \mathrm{~m}$ 以上, 一般 在 $1 \mathrm{~m}$ 左右为多, 短的也有 $20 \mathrm{~cm}$ 左右, 宽 $0.4 \sim 2.0$ $\mathrm{cm}$ 叶顶端钝园, 边缘具不明显疏细齿。苦草花为 雌雄异株, 为典型的水媒传粉方式进行有性繁殖。 在太湖地区，一般 6 月下旬至 11 月下旬是苦草种群 的繁殖期 雄花生长于植株的基部, 在佛焰苞中生长 成熟，大量成熟的雄花脱离总状花序的佛焰苞，漂浮 至水面，且花粉粒端常常朝向水面，花瓣向上像风 帆，借助风力或随水流漂动，传播花粉(图 1a、b、c)。 经调查统计 雄株可以产生 11 33 个佛焰苍, 每个 佛焰苞内有 $311 \sim 412$ 朵雄花, 平均为 $364 \pm 38$ 朵 雄 花能够产生 $128 \sim 184$ 粒花粉, 平均为 $157 \pm 23$ 粒; 因 此,估算被调查的每个雄性植株能够产生花粉粒为 $6.29 \times 10^{5} \sim 1.89 \times 10^{6}$ (表 1 )。雌花单生于一长长的 螺旋状的花茎上 (图 1d), 借助于花茎的伸长, 挺出 水面, 接受漂浮的花粉, 进行授粉。授粉后的雌花闭 合 花茎卷曲收缩入水中。成熟的果实呈长棱形, 长 度 $9 \sim 22 \mathrm{~cm}$, 每个果实生物量平均为 $0.0915 \mathrm{~g} \mathrm{DW}$ (样本数为 100) , 果实内有很多细小的种子 (图 1e), 种子长椭圆形, 长 $0.16 \sim 0.30 \mathrm{~cm}$, 每个果实内含有 的种子多达 $150 \sim 360$ 粒。每 1000 粒种子的生物量 平均为 $0.1238 \pm 0.0038 \mathrm{~g} \mathrm{DW}$ 。根据调查估算, 太 湖苦草种群立地种子量可以达到 $1.68 \times 10^{4} \sim 1.01$ $\times 10^{6}$ 粒 $\mathrm{m}^{-2}$ 。

表 1 苦草雄性植株繁殖生态特征统计

Table 1 Statistical comparison in propagation characteristic of male plants of Vallisneria natans

\begin{tabular}{|c|c|c|c|c|c|c|}
\hline $\begin{array}{c}\text { 样号 } \\
\text { Sample } \\
\text { no. }\end{array}$ & $\begin{array}{l}\text { 花苞数 } \\
\text { Spathe } \\
\text { number }\end{array}$ & $\begin{array}{c}\text { 雄花数/株 } \\
\text { Male flower } \\
\text { number/Ind. } \\
\left(\times 10^{4}\right)\end{array}$ & $\begin{array}{c}\text { 花粉粒数 } \\
\text { Microspore } \\
\text { number }\left(\times 10^{6}\right)\end{array}$ & $\begin{array}{c}\text { 有性繁殖生物量 } \\
\text { Biomass in sexual } \\
\text { propagation (g) }\end{array}$ & $\begin{array}{c}\text { 植株总生物量 } \\
\text { Total biomass } \\
(\mathrm{g})\end{array}$ & $\begin{array}{c}\text { 繁殖部分/总生物量 } \\
\text { Biomass in } \\
\text { propagation/Total } \\
\text { biomass }(\%)\end{array}$ \\
\hline 1 & 33 & 1.20 & 1.89 & 0.18 & 1.30 & 13.8 \\
\hline 2 & 11 & 0.40 & 0.63 & 0.06 & 0.92 & 6.5 \\
\hline 3 & 19 & 0.69 & 1.09 & 0.13 & 1.62 & 8.0 \\
\hline 4 & 31 & 1.13 & 1.77 & 0.17 & 1.11 & 15.3 \\
\hline 5 & 26 & 0.95 & 1.49 & 0.15 & 1.01 & 14.9 \\
\hline 6 & 22 & 0.80 & 1.26 & 0.13 & 1.85 & 7.0 \\
\hline 平均值 Mean & 24 & 0.86 & 1.35 & 0.14 & 1.30 & 10.9 \\
\hline
\end{tabular}

雄花数为每个被调查植株花苞数 $\times$ 花苞内雄花数的平均值, 花粉粒数为每个被调查植株雄花数的平均值 $\times$ 每朵雄花的平均花粉粒数 Male flower number of individual plant $=$ spathe number $\times$ male flower mean number in spathe. Microspore number $=$ male flower number of individual plant $\times$ microspore number in a flower 

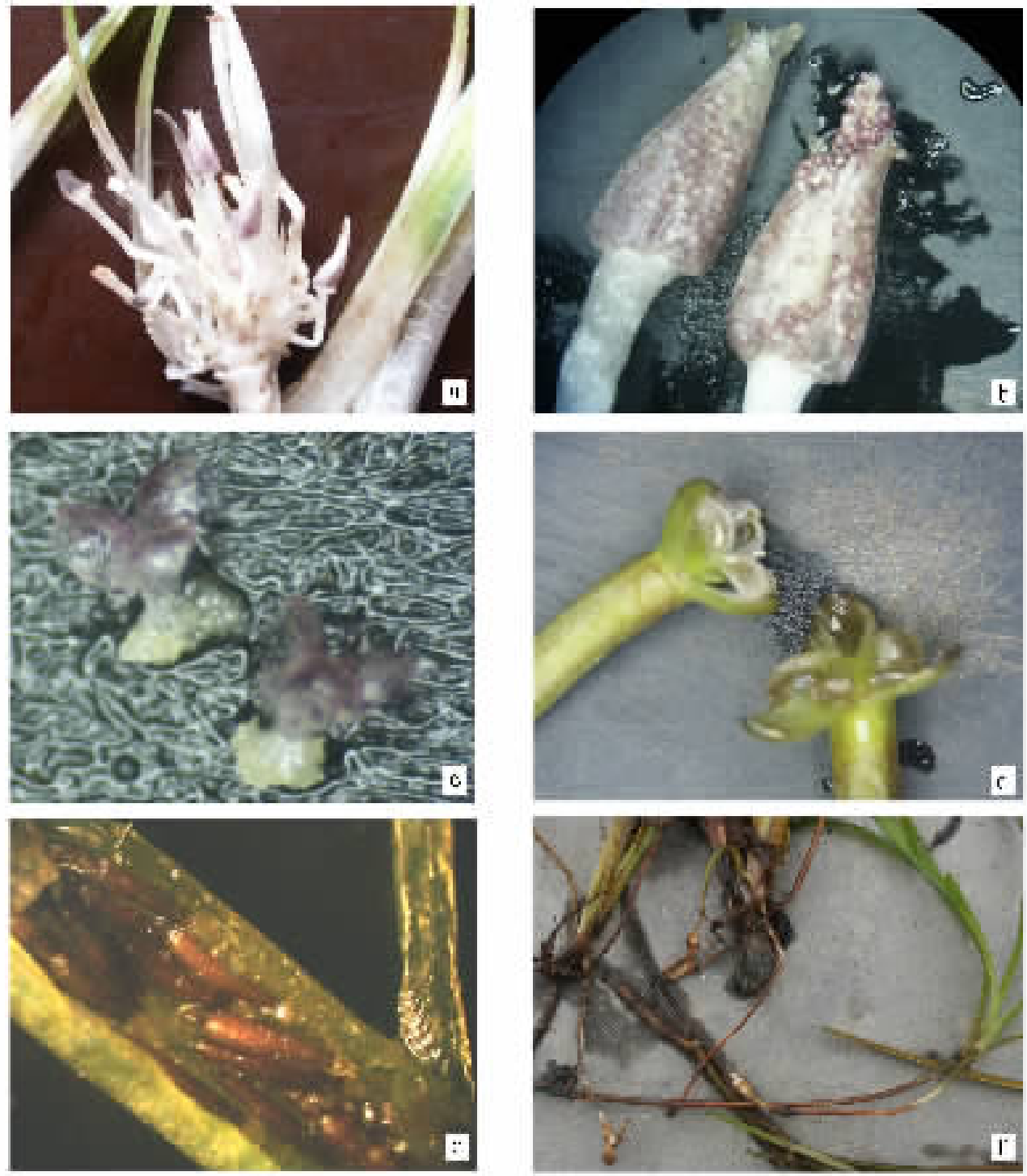

图 1 苦草的有性与无性繁殖部分

Fig.1 Sexual propagule and asexual propagule of Vallisneria natans

a. 生长于基部的雄花 Male flowers b. 雄花苞 Spathe c. 雄花和花粉粒 Male flowers and Microspore d. 雌花 Female flowers e. 种子 Seeds f. 匍匐茎与块茎 Stolon and tubers

调查的所有苦草种群存在有性生殖, 但无性生 殖是其每年种群恢复的主要方式。苦草具匍匐根状 茎, 白色, 于底泥下 $5 \sim 10 \mathrm{~cm}$ 处, 根状茎的茎节处可 以萌发出不定根, 长成新植株。一株苦草母株可以 连续抽发出十几个新植株, 新植株再向四周生长出 横走的根状茎再形成子株。在五里湖围隔试验区观 察发现,一个母株最多可以形成 11 个子株, 匍匐茎 节之间距离为 $2.5 \sim 18.7 \mathrm{~cm}$, 平均为 $7.5 \pm 5.2 \mathrm{~cm}$ 。 每年夏秋季, 苦草植株基部发出的根状茎顶端还能 形成大量长 9 16 mm、宽 3 6 mm 的块茎(冬芽, 也 称球茎, 图 1f)。调查发现 块茎数量为 $90 \sim 226$ 个 $\mathrm{m}^{-2}$ 块茎数量大小依据种群密度而不同, 每个块茎 生物量为 $0.02 \sim 0.11 \mathrm{~g} \mathrm{DW}$, 平均 $0.14 \pm 0.22 \mathrm{~g} \mathrm{DW}$ 。 这些块茎蛰伏于底泥中越冬, 翌年春季萌发成新植 株。每年 3 月，当水温超过 $10{ }^{\circ} \mathrm{C}$ 时 球茎开始萌发， 每个球茎可以萌发 2 3 株新植株; 至 6 月中旬开始 开花结果, 一直到 12 月; 以后, 苦草种群逐渐枯萎。 尽管苦草是多年生沉水植物, 但其种群几乎为一龄 的植株组成。块茎一般存在于 5 30 cm 土层中, 表 层 5 15 cm 深度块茎数量较多, 若按每个块茎总发 芽数计，其发芽率可超过 $100 \%$ 。

苦草的繁殖有 3 种形式，一是种子繁殖，二是通 
过匍匐茎向四周扩展形成子株, 三是通过地下块茎 进行繁殖。苦草分配在繁殖部分的生物量 (按干重 计)较大。调查统计发现(图 2) ,分配在非繁殖部分 的(包括叶片和根)占 $46.6 \% \sim 84.0 \%$ 左右, 平均为 $66.4 \% \pm 12.3 \%$ 繁殖部分占 $16.0 \% \sim 53.4 \%$, 平均 为 $33.6 \% \pm 12.3 \%$, 其中有性部分占 $2.0 \% \sim$ $47.9 \%$, 平均 $25.0 \% \pm 13.8 \%$,无性部分占 $1.1 \%$
$31.4 \%$, 平均 $10.1 \% \pm 7.0 \%$ 。

此外，每个自然株苦草产生的雌花数与座果数 量也较大(表 2), 最多的雌花达 54 个, 平均为 18.9 \pm 11.5 个; 座果率最高达 $100 \%$, 平均为 $71.16 \% \pm$ $15.43 \%$, 实际情况可能要高于表 2 所统计的。野外 调查时发现采集的苦草样品中有些花茎上的果实已 经脱落, 可能被风浪打落或已经成熟而沉入水底。

表 2 苦草雌性植株开花数及座果率

Table 2 Number of female flowers and seedset rate of Vallisneria natans

\begin{tabular}{|c|c|c|c|c|c|c|c|}
\hline $\begin{array}{l}\text { 样品序号 } \\
\text { Sample no. }\end{array}$ & $\begin{array}{c}\text { 开花数 } \\
\text { Flowers number }\end{array}$ & $\begin{array}{c}\text { 座果数 } \\
\text { Seedset number }\end{array}$ & $\begin{array}{l}\text { 座果率\% } \\
\text { Seedset rate }\end{array}$ & $\begin{array}{l}\text { 样品序号 } \\
\text { Sample no. }\end{array}$ & $\begin{array}{c}\text { 开花数 } \\
\text { Flowers number }\end{array}$ & $\begin{array}{c}\text { 座果数 } \\
\text { Seedset number }\end{array}$ & $\begin{array}{l}\text { 座果率\% } \\
\text { Seedset rate }\end{array}$ \\
\hline 1 & 22 & 16 & 72.73 & & & & \\
\hline 2 & 27 & 19 & 70.37 & 19 & 8 & 5 & 62.50 \\
\hline 3 & 5 & 4 & 80.00 & 20 & 17 & 14 & 82.35 \\
\hline 4 & 7 & 6 & 85.71 & 21 & 16 & 11 & 68.75 \\
\hline 5 & 36 & 27 & 75.00 & 22 & 14 & 10 & 71.43 \\
\hline 6 & 2 & 2 & 100.00 & 23 & 22 & 16 & 72.73 \\
\hline 7 & 9 & 8 & 88.89 & 24 & 7 & 5 & 71.43 \\
\hline 8 & 27 & 27 & 100.00 & 25 & 14 & 8 & 57.14 \\
\hline 9 & 9 & 6 & 66.67 & 26 & 26 & 16 & 61.54 \\
\hline 10 & - & - & - & 27 & 20 & 15 & 75.00 \\
\hline 11 & 30 & 11 & 36.67 & 28 & 6 & 5 & 83.33 \\
\hline 12 & 54 & 43 & 79.63 & 29 & 5 & 5 & 100.00 \\
\hline 13 & 24 & 10 & 41.67 & 30 & 31 & 20 & 64.52 \\
\hline 14 & 25 & 16 & 64.00 & 31 & 16 & 11 & 68.75 \\
\hline 15 & 14 & 9 & 64.29 & 32 & 13 & 6 & 46.15 \\
\hline 16 & 30 & 26 & 86.67 & 33 & 22 & 14 & 63.64 \\
\hline 17 & 36 & 22 & 61.11 & 平均值 Mean & 18.91 & 13.09 & 71.16 \\
\hline 18 & 11 & 6 & 54.55 & $S D$ & 11.52 & 8.83 & 15.43 \\
\hline
\end{tabular}
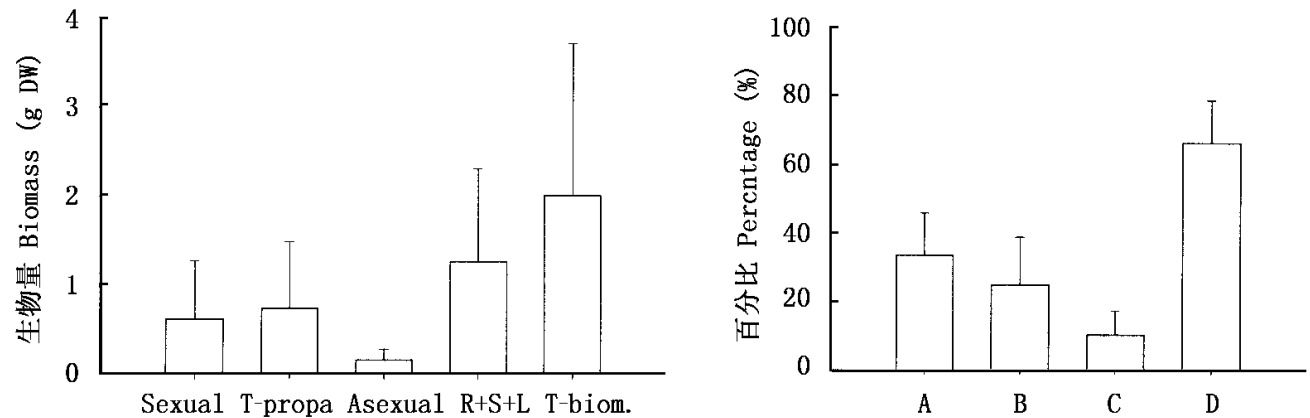

图 2 苦草生物量在各部分分配

Fig.2 Proportion of total propagation , sexual and asexual propagation in biomass of Vallisneria natans

Sexual、T-propa、Asexual、R + S + L、T-biom 分别代表有性繁殖部分、总繁殖部分、无性繁殖部分、根茎叶部分及总生物量 Sexual，T-propa，Asexual and R+S + L show the biomass in part of sexual propagation, asexual propagation, root + shoot + leaf and total biomass , respectively A :(繁殖部分/总生 物量) $\times 100 \%$ (Part of propagation/Total biomass) $\times 100 \%$ B :(有性部分/总生物量) $\times 100 \%$ (Biomass of sexual propagation /Total biomass) $\times 100 \%$ $\mathrm{C}:$ :无性部分/总生物量) $\times 100 \%$ (Biomass of asexual propagation/Total biomass) $\times 100 \%$ D :(根茎叶部分/总生物量) $\times 100 \%$ (Biomass of Root + shoot + leaf/Total biomass) $\times 100 \%$ 


\section{2 种子发芽试验}

室内种子发芽试验共进行了 $75 \mathrm{~d}$, 到试验结束 时, 10、20、30 ${ }^{\circ} \mathrm{C}$ 恒温条件下, 保存 4 个月的种子发芽 率分别为 $8.35 \% \pm 1.89 \% 、 56.73 \% \pm 6.42 \%$ 、 $43.55 \% \pm 4.34 \%$ 。可以看出, $10{ }^{\circ} \mathrm{C}$ 时的发芽率最 低, 与其它两组比较差异极显著 $(p<0.01)$, 最高为 $20{ }^{\circ} \mathrm{C}, 30{ }^{\circ} \mathrm{C}$ 时发芽率有所下降 (图 3 左) 20 与 $30{ }^{\circ} \mathrm{C}$ 间差异显著 $(p<0.01)$ 。

苦草种子发芽对基质有一定要求。从图 3(左) 中可以看出, 在没有湖泥的条件下, 发芽率为 $36.02 \% \pm 2.58 \%$, 比有湖泥的下降 $36.5 \%(p<$ 0.01 )。

变温对最终的发芽率影响不大, 在有湖泥、30/ $10 、 20 / 10{ }^{\circ} \mathrm{C}$ 变温条件下的发芽率分别为 $59.13 \% \pm$ $4.44 \%$ 和 $56.72 \% \pm 5.09 \%$, 两组间差异不显著 $(p>$ $0.05)$ 。但是 $30 / 10{ }^{\circ} \mathrm{C}$ 变温可以增加种子发芽速率, 在此处理下 发芽历期仅 $20 \mathrm{~d}$ 左右。

野外现场观察发现，苦草种子不存在休眠，所采 集的当年种子中就已有发芽的。从试验结果看 (图 3 右) 未脱水、保存期短的当年种子发芽率最高, 为 $56.73 \% \pm 6.42 \%$ (图 3 左), 干燥保存 $1 、 2$ 年的种子 发芽率分别为 $35.53 \% \pm 3.53 \%$ 和 $12.63 \% \pm$ $2.86 \%$ ，两组间差异极显著 $(p<0.01)$; 干燥保存 4 年的种子仅发现 2 粒种子发芽 继续观察了 $20 \mathrm{~d}$, 其 结果相同。表明随干燥保存时间增加，其发芽率降 低 $A$ 年以上的种子已经基本不发芽。

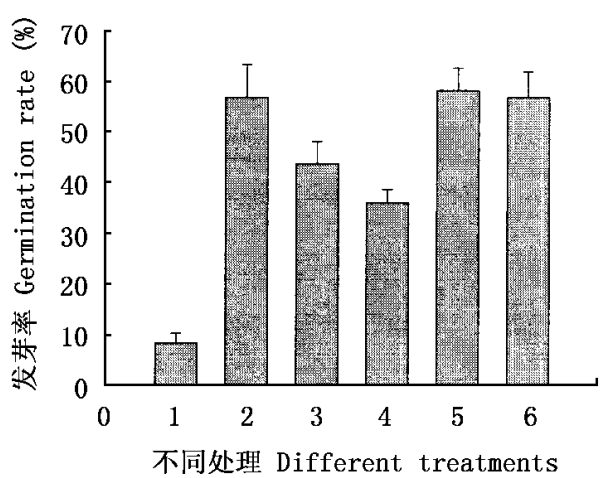

图 3 不同处理组的苦草种子发芽率

Fig.3 Germination rate in seeds of different treatments of Vallisneria natans

左图为当年种子的发芽率, 图中 $1 、 2 、 3$ 分别代表 $10 、 20 、 30{ }^{\circ} \mathrm{C}$ 条件下的发芽率 $A$ 代表无湖泥的 $20{ }^{\circ} \mathrm{C}$ 条件下的发芽率 5 和 6 分别代表有湖 泥的 $30 / 10$ 和 $20 / 10{ }^{\circ} \mathrm{C}$ 变温条件下的发芽率 ;右图为有湖泥、 $20{ }^{\circ} \mathrm{C}$ 条件下的发芽率 其中 1 代表干燥保存 1 年的种子在有光下的发芽率 , 2 代表 干燥保存 1 年的种子在无光下的发芽率, 3 和 4 分别代表干燥保存 2 和 4 年的种子在有光下的发芽率 Left figure indicates germination rate of 4 months conserved seeds. $1-3$ show germination rates of seeds at $10,20,30{ }^{\circ} \mathrm{C}$ with lake sediment , respectively. 4 shows germination rate of seeds at $20{ }^{\circ} \mathrm{C}$ without lake sediment. 5 and 6 show germination rates of seeds under conditions of $30 / 10,20 / 10{ }^{\circ} \mathrm{C}$. Right figure indicates germination rate at $20{ }^{\circ} \mathrm{C}$ with lake sediment. 1 shows germination rate of 1 -year conserved seeds at $20{ }^{\circ} \mathrm{C}$ with natural light. 2 shows germination rate of 1-year conserved seeds at $20{ }^{\circ} \mathrm{C}$ without natural light. 3 and 4 show germination rates of 2-year conserved seeds and 4-year conserved at $20{ }^{\circ} \mathrm{C}$ with natural light 


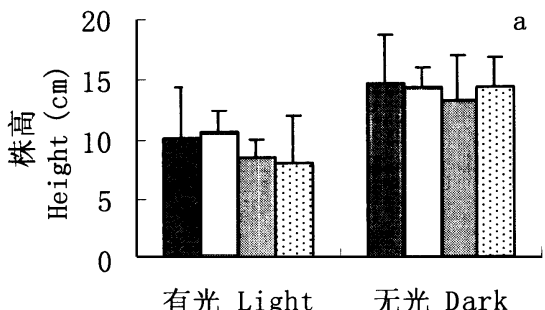

有光 Light 无光 Dark
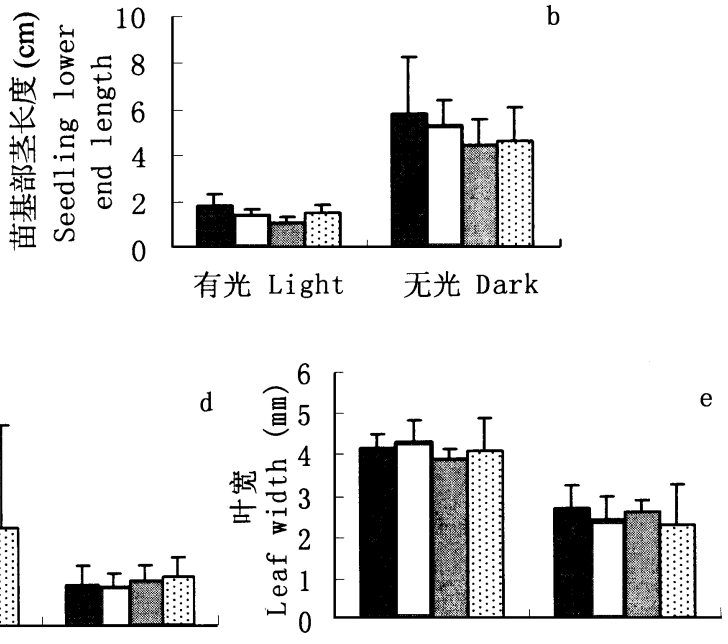

有光 Light 无光 Dark

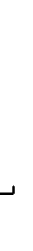

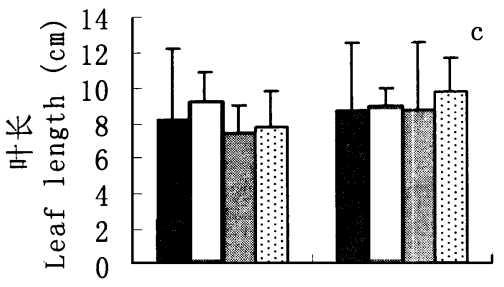

有光 Light 无光 Dark

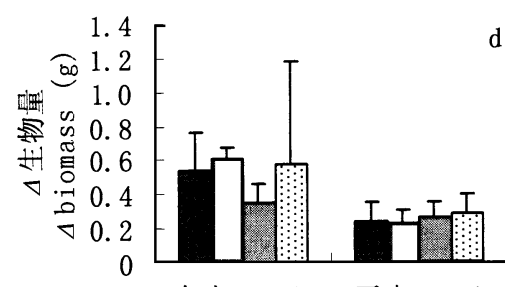

有光 Light 无光 Dark

口组1 Group 1 口组2 Group 2 组3 Group 3 曰组4 Group 4

图 4 在有光和无光条件下苦草块茎苗生长的差异

Fig.4 Impact of light on seedlings reproduced from root tuber of Vallisneria natans

从苦草的块茎苗在有、无光条件下的生长情况 看(图 4a、b、c) , 有光条件下经过 $15 \mathrm{~d}$ 的生长, 幼苗 高度平均为 $8.41 \pm 1.56 \sim 10.56 \pm 1.74 \mathrm{~cm}$, 平均生 长速度为 $0.56 \sim 0.70 \mathrm{~cm} \mathrm{~d}^{-1}$, 而无光条件下的苗高 平均为 $12.97 \pm 3.78 \sim 14.41 \pm 4.08 \mathrm{~cm}$, 平均生长速 度为 $0.86 \sim 0.96 \mathrm{~cm} \mathrm{~d}^{-1}$, 差异极显著 $(p<0.01)$; 从 幼苗的茎长看, 有光的苦草莲座叶基部的根茎长度 平均为 $1.10 \pm 0.25 \sim 1.83 \pm 0.49 \mathrm{~cm}$, 无光的平均为 $4.40 \pm 1.1 \sim 5.74 \pm 2.38 \mathrm{~cm}$, 有光的比无光的低 $68 \% \sim 75 \%$ 差异显著 $(p<0.01)$; 幼苗的叶长看, 有光的为 $7.36 \pm 1.61 \sim 9.14 \pm 1.72 \mathrm{~cm}$, 无光的为 $8.57 \pm 3.82 \sim 9.64 \pm 1.88 \mathrm{~cm}$, 差异不显著 $(p>$ $0.05)$, 这 3 方面结果表明, 有光条件的伸长生长较 无光条件下慢, 块茎苗初期的伸长生长主要依赖于 基部的根茎生长。

从图 $4 \mathrm{e}$ 中可以看出, 有光的幼苗叶宽平均为 $3.89 \pm 0.24 \sim 4.31 \pm 0.49 \mathrm{~mm}$, 无光的平均为 $2.20 \pm$ $1.00 \sim 2.63 \pm 0.60 \mathrm{~mm}$, 差异极显著 $(p<0.01)$, 而叶 的长度差异不显著 (图 4c), 表明有光条件下其植株 生长较粗壮。此外, 图 $4 \mathrm{~d}$ 结果显示, 苦草球茎苗在 有光条件下生物量积累较无光的大, 表明在有光条 件下苦草生长速度快, 这主要是因为幼苗生物量的 积累来自自养和异养两个部分。从试验结果看, 无 光条件下植株拔高较快, 这是水生植物自身适应弱 光环境的自我调节机制, 这种适应机制可以使沉水 植物发芽后迅速达到水下有效光合层, 从而使其异 养生长迅速转为自养光合生长。

\section{3 讨 论}

通过对太湖的野外调查发现, 苦草在繁殖部分 的投资较其它沉水植物大得多, 繁殖部分占植株总 生物量 (干重)达到 $16.0 \%$ 53.4\% , 平均为 $33.6 \%$ $\pm 12.3 \%$, 其中有性部分大于无性部分, 分别占 $2.0 \% \sim 47.9 \%$ (平均 $25.0 \% \pm 13.8 \%$ ) 和 $1.1 \%$ $31.43 \%$ (平均 $10.1 \% \pm 7.0 \%$ )。虽然苦草有 3 种繁 殖方式 (种子、匍匐茎和块茎)，但每年种群恢复与发 展主要依赖地下块茎和匍匐茎, 调查期间很难找到 种子苗。那么, 对于湖泊、水库等大水体而言, 苦草 在有性部分的大量资源投资是非常浪费的。根据太 湖调查估算, 苦草种群立地种子量可以达到 $1.68 \times$ $10^{4} \sim 1.01 \times 10^{6}$ 粒 $\mathrm{m}^{-2}$, 而成熟的种子是沉水性的， 不易随水流漂移，对种群扩张也贡献不大。

Donnermeyer 和 Smart (1985) 研究发现, 苦草 $(V$. americanna) 在繁殖部分的投资占总生物量 (干重)的 $30 \%$ 其中有性繁殖体占 $5 \%$ 左右, 无性繁殖体占 $25 \%$ 左右。繁殖部分占总生物量比值与我们调查的 结果相似, 但有性与无性繁殖体所占总生物量比例 差异较大, 这可能与种类或所处的环境条件不同有 关。在较深水域生长的苦草需要较大营养生长来积 累干物质 将会影响这一比值。

苦草地下块茎数量比较丰富, 是苦草种群恢复 的强大物质基础, 但对其报道差异较大。朱海虹和 张本 (1997) 等在鄱阳湖的子湖——蚌湖西南部 (芦 潭村东北) 苦草分布区的调查报道, 苦草冬芽密度为 
5 个 $\mathrm{m}^{-2}$ 。熊秉红和李伟 (2000) 对鄱阳湖自然保护 区 2 个子湖——蚌湖和中湖池的调查显示,生活冬 芽在 2 个湖泊中的平均密度分别为 15.8 个 $\mathrm{m}^{-2}$ 和 24.3 个 $\mathrm{m}^{-2}$ 。然而笔者在野外调查时发现, 苦草地 下块茎要远大于文献所报道的, 达到 $90 \sim 226$ 个. $\mathrm{m}^{-2}$ 。造成差异的因素与苦草种群密度、环境条件 及采样方法有关。苦草的块茎在地下埋藏较深, 调 查时需要用柱状采泥器进行高密度采集表层 $40 \mathrm{~cm}$ 以上的泥样, 淘洗计数。

从种子发芽试验结果可以看出, 种子在温度为 $20{ }^{\circ} \mathrm{C}$ 时发芽率较高, 需要有一定底泥, 而且, 变温较 大的处理组萌发率高, 萌发速率也快, 发芽历期短。 变温大的条件促进水生植物种子发芽在湿地植物类 群中较为常见 (Lombardi et al.,1997; Brenchley \& Probert ,1998; Smolders et al. ,1995)。光照对苦草种 子发芽也有较大的影响, 保存 1 年的种子在无光条 件下其发芽率较有光的下降了 $63.6 \%$ ，一般水生植 物中小种子类群发芽对光有需求 (Grime et al. , 1981 ; Thompson \& Grime, 1983)。此外, 种子保存时 间对发芽率影响较大, 当年种子 (湖泊流水环境保存 4 个月左右) 发芽率最高, 干燥保存时间越长, 其发 芽率越低,干燥保存 4 年的种子已经几乎不发芽。 因此，在恢复湖泊沉水植被时，或利用种子培植苦草 来进行渔业生产时, 应注意在水温 $20{ }^{\circ} \mathrm{C}$ 度左右、利 用前一生长季的苦草种子来进行培植与恢复苦草种 群 培植时应在浅水(水深小于 $50 \mathrm{~cm}$ 较适宜)、相对 稳定的水域进行, 否则, 因种子苗非常纤细, 易被风 浪摚起漂浮, 难以定植成功。此外, 需要强调是, 笔 者经过多年努力, 在湖泊或深水池塘中利用种子来 恢复苦草种群均未成功, 仅在浅水池塘获得较好效 果。

块茎发芽试验表明, 块茎萌发率相当高, 在水温 为 $20{ }^{\circ} \mathrm{C}$ 下, 可达 $90.2 \% \pm 12.6 \%$,无光条件对其发 芽率影响也不大。李文朝和连文华 (1995) 指出, 苦 草冬芽的萌发无需光刺激, 但要使新生苗形成足够 的叶绿素, 仍然需要一定的光照条件, 与本试验结果 一致。他同时指出, 苦草冬芽萌发后的相对光照强 度 $I / I_{\mathrm{air}}$ 小于 $5 \%$ 时能引起幼苗白化和光合作用受 阻，但没有报道在无光照条件下幼苗自养生长可以 达到的高度, 而在无光下幼苗依赖异养生长是否能 够达到水体有效光合层而成功进入自养生长阶段是 苦草种群每年恢复的关键, 从本研究结果看 (图 4a) 苦草依靠自身积累的营养进行异养生长可以达 到 $15 \mathrm{~cm}$ 高度。
根据文献, 苦草球茎的萌发受土层厚度的影响 很大, 被 $5 \mathrm{~cm}$ 土层覆盖时萌发率为 $100 \%$; 俚层厚度 为 $15 \mathrm{~cm}$ 时萌发率为 $73.3 \%$;土层厚度为 $25 \mathrm{~cm}$ 时 萌发率仅为 $3.3 \%$ (熊秉红和李伟, 2000)。因此, 在 利用球茎培植苦草时, 应注意埋藏深度。

另据试验结果, 有光条件下的苦草块茎苗伸长 生长比无光条件下缓慢, 块茎苗初期的伸长生长主 要依赖于基部的根茎生长。块茎苗在有光条件下生 物量积累比无光组的大, 这主要是因为幼苗生物量 的积累来自自养和异养两个部分。但无光条件下植 株拔高较快, 这是水生植物自身适应弱光环境的自 我调节机制, 这种适应机制可以使沉水植物发芽后 迅速达到水下有效光合层, 从而使其异养生长迅速 转为自养光合生长。

本试验未能就不同水位、更多不同温度分级对 种子发芽影响作更细致的研究, 以及苦草发芽的生 物学积温, 这些方面可为渔业养殖提供理论上的技 术指导,并为湖泊生态修复提供更深入的理论依据， 在以后的研究工作中是值得探讨的。

\section{参 考 文 献}

Brenchley JL, Probert RJ (1998). Seed germination responses to some environmental factors in the seagrass Zostera capricorni from eastern Australia. Aquatic Botany, 62, 177 - 188 .

Donnermeyer GN, Smart MM (1985). The biomass and nutritive potential of Vallisneria americana Michx. in navigation pool 9 of the Upper Mississippi River. Aquatic Botany, 22, 33-44.

Grime JP, Mason G, Curtis AV, Rodman J, Band SR, Mowforth MAG, Neal AM, Shaw S (1981). A comparative study of germination characteristics in a local flora. Journal of Ecology, 69, 1017 - 1059 .

Gu LD (顾林娣), Chen J (陈坚), Zhang ZS (章宗涉) (1994)。 Effect of cutting on growth on Vallisneris spiralis. Plant Physiology Communications (植物生理学通讯), 30, 105 - 107. (in Chinese)

Li YD (李亚东), Cui YQ (崔艳秋) ( 2000). Germination experiment on seeds and stem tubers of Vallisneria natans in Lake Donghu of Wuhan. Acta Hydrobiologica Sinica (水生生物学 报), 24, 298-300. (in Chinese with English abstract)

Li WC (李文朝), Lian GH (连文华) (1995). Light demand for brood-bud germination of submerged plant. Journal of Lake Sciences (湖泊科学), 7(Suppl.), 25-27. (in Chinese with English abstract)

Lombardi T, Fochetti T, Bertacchi A, Onnis A (1997). Germination requirements in a population of Typha latifolia. Aquatic Botany, 56, $1-10$.

Smolders AJP, den Hartog C, Roelofs JGM (1995). Germination and seedling development in Stratiotes aloides L. Aquatic 
Botany, 51, $269-279$.

Song F (宋福), Chen YQ (陈艳卿), Qiao JR (乔建荣), Ren JC (任久长) (1997). Study on the removal rate to total nitrogen in Caohai Lake (including sediments) by common submerged macrophytes. Research of Environmental Sciences (环境科学研 究) , 10(4), 47-50. (in Chinese with English abstract)

Sun XZ (孙祥钟) (1992). Flora (Tomus 8) (中国植物志, 第 8 卷). Science Press, Beijing, 177 - 179. (in Chinese)

Thompson K, Grime JP (1983). A comparative study of germination responses to diurnally-fluctuating temperatures. Journal of Applied Ecology, 20, 141 - 156.

Xiong BH (熊秉红), Li W (李伟) (2000). Ecological studies on Vallisneria L. in China. Journal of Wuhan Botanical Research
(武汉植物学研究) , 18, 500-508. (in Chinese with English abstract)

Yan SZ (颜素珠) (1983). Illustrated Handbook in Chinese Aquatic Macrophytes (中国水生高等植物图说). Science Press, Beijing, $237-239$. (in Chinese)

You WH (由文辉), Song YC (宋永昌) (1995). Seed germination ecology of three submerged macrophytes in Dianshan Lake. Chinese Journal of Applied Ecology (应用生态学报)，6, 196 200. (in Chinese with English abstract)

Zhu HH (朱海虹), Zhang B (张本) (1997). Poyang Lake (鄱 阳湖). University of Science and Technology of China Press, Hefei, 205 - 206. (in Chinese) 